\title{
DERRIDA Y LA TRAGEDIA DE LA REPRESENTACIÓN
}

\section{DERRIDA AND THE TRAGEDY OF REPRESENTATION}

\author{
Juana Isabel López BERNAL \\ UNED
}

\begin{abstract}
Resumen: El presente trabajo realiza una lectura de "Le sacrifice", trascripción de una intervención realizada por Jacques Derrida el 20 de octubre de 1991 en el Teatro Nacional de Lille, en el curso de un encuentro titulado: L'irreprésentable, le secret, la nuit, le forclos. Fue publicado conjuntamente por primera vez en 1993 por la revista $L a$ Métaphore, de Éditions de la Différence y la revista Lieux extrêmes. En este texto Derrida nos propone una reflexión sobre el teatro de Daniel Mesguich (Argel, 1952), autor, director de escena de teatro y ópera, actor de cine y teatro, profesor y traductor. Nuestro texto se inscribe en los pliegues de esta lectura de Derrida, en su reflexión sobre la tragedia, el sacrificio y el tiempo del teatro, realizando así una nueva lectura que incluirá en su camino a Sófocles, Platón, Freud, María Zambrano... A través de esta lectura de Derrida nos aproximamos a la representación en el teatro para, desde ahí, abordar otros contextos que también están presentes en el texto: desde la representación lingüística hasta el concepto psicoanalítico de la representación que incorpora la represión para acabar destacando también, de la mano de Antígona, cierta crítica al falogocentrismo.
\end{abstract}

Palabras ClaVe: Deconstrucción, teatro, representación, tragedia, represión.

Aвstract: This paper provides a reading of "Le Sacrifice", a transcription of Jacques Derrida's speech at the conference Lirreprésentable, le secret, la nuit, le forclos at the Lille National Theatre on 20th October 1991. This speech was first published jointly by the magazines La Métaphore (Editions La Différence) and Lieux extremes in 1993. In this text, Derrida reflects on the theatre of Daniel Mesguich (Algeria, 1952), author, theatre and opera stage director, theatre and cinema actor, professor, and translator. Our work is inscribed in the folds of this reading of Derrida, in his reflection on tragedy, 
sacrifice and the time of theatre, thus carrying out a new reading that includes Sophocles, Plato, Freud, María Zambrano... Through this reading of Derrida, we get closer to representation in the theatre in order to, from there, approach other contexts which are also present in the text: from linguistic representation to the psychoanalytic concept of representation that incorporates repression, concluding by emphasizing, through Antigone, a kind of criticism of phallogocentrism.

KeYwORDs: Deconstruction, theatre, tragedy, representation, repression.

\section{Introducción}

El teatro y la representación son el núcleo temático de esta reflexión que nos propone Derrida en este pequeño texto que es la trascripción de una intervención realizada en un encuentro acerca del teatro. Por lo tanto, es la representación escrita de una intervención hablada, (representada en el teatro) que fue en primer lugar una intervención escrita. Y aunque esto parecería circunscribir nuestro enfoque hacia una perspectiva lingüística, nada más lejos de nuestra intención. La extensión del campo textual, el despliegue de la textualidad sobre otros contextos de representación ha sido una de las labores que la estrategia deconstructiva de Jacques Derrida ha realizado desde sus primeros trabajos. El teatro y el concepto de representación que el pensamiento clásico del mismo incorpora son un elemento fundamental para esta lectura. Derrida se ha servido de la crítica a ese concepto clásico de representación en el teatro que no deja de tener una raigambre filosófica, metafísica, una trama platónico-aristotélica ${ }^{1}$. Ese concepto de representación supone siempre que hay una realidad anterior subyacente, única y singular, que sirve como modelo a esa secundaria realidad. Pero en el campo de lo teatral, la representación es también representación de ideas a través de la imagen, transmisión de mensajes cifrados, metáforas visuales y fónicas que conmueven. Hay una intencionalidad de la representación teatral y en el análisis de la tragedia griega, como género literario y como arte dramático, escenográfico, esa intencionalidad también es ética y política. El pensamiento que nos propone Jacques Derrida de lo teatral, del teatro, se inserta en la reflexión realizada por Mesguich. Pero esta reflexión deconstructiva debe situarse en el contexto más amplio del pensamiento de la representación como iterabilidad, como repetición inaugural de un acontecimiento singular, único, al que dota de

${ }^{1}$ Sobre el concepto de representación, cfr. "Le retrait de la métaphore" y "Envoi" en Derrida, J. (1987): Psyché. Inventions de l'autre. Paris: Galilée. 
esta característica el hecho de poder ser repetido. Esta idea de iterabilidad que hace nacer una nueva representación marca también una distinta perspectiva del tiempo. La representación teatral alumbra una temporalidad que cuestiona el presente. Desde esa nueva perspectiva, y teniendo en cuenta las reflexiones que Daniel Mesguich realiza en su obra L'éternel éphémère (1991), Derrida nos va a proponer una visión imposible, la de aquello que es irrepresentable, que no se deja o no se puede representar, bien porque escapa al ámbito de lo visible bien porque aunque visible, carecemos de la posibilidad de interpretarlo como tal y por lo tanto, forma parte de lo excluido, del secreto no conocido a veces ni por aquel que lo guarda. $Y$ en ese contexto, de la mano de Antígona nos acercaremos a la representación también de la mujer, su papel entre la familia y la ley, a la representación de la mujer como lo excluido también del pensamiento, y por lo tanto, al falogocentrismo que desde sus primeros textos Derrida ha venido señalando como uno de los pilares del edificio de la metafísica.

\section{La palabra invisible}

Que la filosofía y el teatro comparten el privilegio otorgado a la visión y a la presencia: ésta es la afirmación con la que comienza Le sacrifice (Derrida, 1993). De este modo dicho parecería que tal afirmación formase parte del ritual y que se sacrificase en este acto al resto de sensaciones, a aquellas que no provengan de la visión de la presencia, del acto presente representado mediante una visión objetivadora. Sin embargo, el crédito dado a este mirar, que es sólo un determinado modo de mirar (que no de ver), acabará por limitar tanto a la filosofía como al teatro, adscribiéndolos a lo que aquí es denominado como historia de la visibilidad, a una cierta interpretación de lo visible. Pero en esta interpretación lo invisible, la visibilidad de lo visible, aquello que hace que algo sea visible pero que sin embargo no es visible, no se ha tenido en cuenta. Se trataría entonces de, como afirma Derrida, dejar un lugar «(...) a lo invisible en el corazón de lo visible, a lo no teorizable en el corazón de lo teórico, a lo no teatral, como un golpe de efecto, en el corazón del teatro» (53).

Sin embargo, hay en esta representación de la visibilidad, de lo invisible de lo visible, un aspecto que no pertenece y que al mismo tiempo forma parte de esa historia de la visibilidad. Es esa "palabra por esencia invisible, venida de debajo de lo visible» que Derrida ha unido a la visibilidad de lo visible, palabra a la que esta visibilidad deja su lugar «en la representación de sí, en la repetición de sí» (53). De este modo encontramos no sólo lo invisible en el corazón de 
lo visible ${ }^{2}$, sino también lo audible en el corazón de lo visible. Y este aspecto puede destacarse tanto en la filosofía como en el teatro.

Que la filosofía también tiene oídos es algo que ya Derrida ha destacado en otro trabajo (1994) donde se pone de manifiesto que, finalmente, la filosofía oye del mismo modo que ve, y que es imprescindible analizar «la complicidad circular de la metáfora del ojo y del oído» (19). De este modo, la palabra invisible, ya sea como voz, ya como texto escrito -y por lo tanto visión- está trabajando tanto en el teatro como en la filosofía, en la metáfora de la visión, e incluso en la visión misma.

Parece pues que la filosofía no ha sabido mirar y oír más que bajo la metáfora cognoscitiva del ojo que privilegia la presencia. Hay, por lo tanto, que mirar de otra manera, que oír de otra manera, que escribir de otra manera si no queremos quedarnos en ese estrecho margen, no suficientemente reflexionado en sus limitaciones, que proporciona esta metáfora. Porque el límite -que es un límite visual- inscribe a la filosofía en una reflexión que es finalmente una reflexión sobre sí misma. Del mismo modo que la representación teatral acaba siendo una representación, cada vez, del teatro mismo. Tanto filosofía como teatro se muestran a sí mismas en su respectivo actuar.

Hay algunos momentos en la filosofía en los que ésta aparece teñida de un cierto halo teatral. Un halo que convierte algunos pasajes de obras filosóficas emblemáticas en ejemplos que nos podemos imaginar como si de obras de teatro se tratase. La obra de Platón es muy fácil de pensar en esta forma debido a su estructura en forma de diálogo. De ella podemos extraer argumentos para numerosas obras de teatro. Un ejemplo extraído de La República:

El escenario representa un antro subterráneo que tiene en toda su anchura una abertura que da paso libre a la luz. En este antro se encuentran hombres encadenados desde su infancia de tal manera que no pueden cambiar de lugar ni mover la cabeza a causa de las cadenas que les sujetan las piernas y el cuello, pudiendo ver solamente los objetos que tienen enfrente. Detrás de ellos, a cierta distancia y a cierta altura, un fuego cuyo resplandor los alumbra, y un camino elevado entre este fuego y los cautivos. A lo largo de

\footnotetext{
${ }^{2}$ Sobre la cuestión de la visibilidad y la ceguera en el pensamiento, en el lenguaje, en la representación, cfr. DERRIDA, Jacques (1990): Mémoires d'aveugle. L'autoportrait et outres ruines, Paris, Louvre, Réunion des Musées Nationaux.
} 
ese camino un tabique, semejante a la mampara que los titiriteros ponen entre ellos y los espectadores. Unas personas pasan a lo largo del tabique llevando objetos de toda clase, figuras de hombres, de animales de madera o de piedra, de suerte que todo esto sobresale del tabique. Entre los portadores de estas cosas unos irán hablando y otros pasarán sin decir nada. (518c-d)

La escenografía está planteada. Ahora tan sólo nos queda hacer dialogar a los personajes. Unos esclavos condenados a una visión única y cuyo conocimiento, porque tienen conocimiento, se encuentra limitado, por tanto, a la asociación de sombras y palabras. Podemos imaginar a estos esclavos utilizando las palabras de Segismundo en La vida es sueño de Calderón. Al igual que en esta obra, un encadenado será rescatado de la oscuridad de su cautiverio, llevado a la luz, y posteriormente sacrificado, obligado a bajar de nuevo a ese antro oscuro y frío sin la luz del sol, y que ahora, con las pupilas reducidas, es aún más lúgubre y oscuro. El sacrificio de este esclavo se hace de modo ilustrativo. Con este sacrificio, sin el cual Platón no puede establecer su idea de la contemplación del Bien, la filosofía toma cuerpo. Nos interesa destacar este ejemplo puesto que, como señala Derrida, se ha establecido la idea de que con la aparición del pensamiento filosófico se puso fin a la tragedia griega. Si la filosofía ha sepultado a la tragedia, al mito y a lo que representa, tal vez lo haya hecho dentro de sí misma. Tal vez no se trate de un final sino de un ocultamiento (represión). Quizá esa estructura del sacrificio, esa contradicción que representa la tragedia y la intensidad dramática que muestra, como más adelante dirá Derrida, recorra toda la filosofía. La explicación y la reflexión de Platón en torno a la contemplación de la idea de Bien tienen un carácter exclusivamente visual, que se manifiesta en su conclusión:

(...) cada cual tiene en su alma la facultad de aprender mediante un órgano destinado a este fin; que todo el secreto consiste en llevar este órgano, y con él el alma toda, de la vista de lo que nace a la contemplación de lo que es, hasta que pueda fijar la mirada en lo más luminoso que hay en el ser mismo, es decir, según nuestra doctrina, en el bien; en la misma forma que si el ojo no tuviere un movimiento particular, sería necesario que todo el cuerpo girase sobre él al pasar de las tinieblas a la luz (...) (Rep., 518c-d)

La autoridad de la mirada, la metáfora visual inaugura también el espacio filosófico. Pero esta autoridad no es privilegio de la filosofía. En la tragedia griega encontramos también un tratamiento teatral de la metáfora del ojo que identifica el conocimiento con la visión. En Edipo rey de Sófocles encontramos el elemento visual analizado de una manera paradójica. Edipo, en su intento de 
salvar a la ciudad, llama al ciego, pero vidente, Tiresias. A pesar de que éste es ciego ve claramente el destino de Edipo, un destino al que Edipo, en un primer momento, es ciego. Las palabras del adivino expresan su visión del problema de Edipo. Tiresias ve. El enojo de Edipo ante la falta de respuesta de Tiresias es tal que estalla y en su cólera provoca al ciego hasta que éste, ofendido, le cuenta el fatal desenlace que tendrá su vida. Edipo no da crédito a las palabras de Tiresias y le acusa de ser el asesino de Layo (del que todavía desconoce Edipo que es su padre). Ante esta acusación Edipo recrimina a Tiresias echándole en cara su ceguera, y algo más: «(...) porque eres ciego en tus oídos, en tu mente y en tus ojos.» (371-372)

La visión, pues, no tiene que ver con el ojo únicamente. La metáfora de la ceguera se hace extensible a todos los sentidos, quedando privilegiado en ese uso la primacía del carácter cognoscitivo de la mirada. Del mismo modo, la existencia de la visión no es suficiente para ver. Edipo ve, pero Tiresias, ante las palabras de Edipo, le responde: «Y te digo, puesto que ahora me ultrajaste de ciego, que tú tienes vista y no ves en qué punto de desgracia estás, ni dónde habitas, ni con quiénes vives.» (412-414)

Cuando Edipo conoce su verdadero origen y su doble crimen, matar a su padre y desposar a su madre, se salta los ojos ante el cadáver de Yocasta, su madre y esposa. Ahora Edipo es ciego pero ve, ahora está igual que el anciano Tiresias. Edipo se ciega porque, ahora que ha visto, no quiere seguir viendo, porque ahora, al conocer la magnitud de sus crímenes - de los que no es responsable pero de los que tiene que responder-, no podría mirar a nadie, lo que convierte a la mirada al otro en un acto con una dimensión ética. Así exclama Edipo:

Yo no sé con qué ojos, en caso de que siguiera con vista, a mi padre podría mirar cuando llegase al Hades, ni de igual forma a mi desventurada madre, contra los que por mi mano hay hechas acciones superiores en su castigo a la horca. ¿Pero es que podía haber en mí sin duda el deseo de contemplar el espectáculo de mis hijos, habiendo brotado como han brotado? En modo alguno, al menos para mis ojos. Ni la ciudad ni torre alguna ni las sagradas estaturas de los dioses, de todo lo cual, yo, desdichado, que la mejor vida de todas, al menos en Tebas, llevé, me privé a mismo, puesto que fui yo quien ordenó que todo el mundo rechazase al impío, al que conforme a los dioses se reveló impuro y de la estirpe de Layo. ¿’Tras descubrir yo mía tal mancha, había de poder mirar a éstos con rectos ojos? (1384-1385) 
La metáfora del ojo no es una metáfora más. Es la metáfora por excelencia. Es la que instituye el conocimiento. Pero esta metáfora se encuentra atrapada, ya desde el principio, por sus propias aporías. Aquellas que provienen de la propia estructura del ojo, de su fisiología, de sus limitaciones. La metáfora del ojo se complica en la actualidad con la técnica. La virtualidad de la visión en nuestros días, los numerosos mecanismos ópticos que funcionan a modo de ojos proporcionan una nueva visión. Visión peligrosa si no se pone en cuestión la metáfora originaria, si no se hace temblar la asociación vista/conocimiento. Y ahí la deconstrucción tiene mucho que ver.

\section{EI sacrificio}

De todas las consideraciones realizadas por Mesguich en su obra L'éternel éphémère, Derrida encuentra muy sugerente la que realiza en torno al sacrificio. En primer lugar se destaca que la filosofía ha puesto fin a cierta tragedia. Por otro lado, que el sacrificio es constitutivo de la tragedia, del espacio trágico. ¿Ha sacrificado la filosofía a la tragedia? "Lejos de que la filosofía haya puesto fin al sacrificio, o justamente porque ha creído ahí poner fin a la tragedia griega, ésta no habría hecho más que portar en sí, bajo otra forma, la estructura sacrificial.» $(1993,57)$ Derrida analiza las dos tesis expuestas a este respecto por Mesguich. La primera de ellas nos dice que la tragedia no tiene lugar en el teatro, pero está puesta en juego (mise en jeu). No hay un presente de la tragedia en el teatro. Para establecer esta tesis Derrida juega con la distinción que realiza Mesguich entre dos tipos de acontecimientos: uno como tener lugar y otro como puesta en juego (en escena). Para Mesguich: «La verdadera tragedia no tiene jamás lugar en el teatro. En el teatro la tragedia es puesta en juego (representada)» (Derrida, 1993, 57). Que la tragedia no tiene lugar en el teatro vendría a decirnos también que ésta no es constitutiva del teatro, que la esencia del teatro no es la tragedia. Pero de la afirmación de Mesguich también parece desprenderse la idea de un verdadero sacrificio, un acontecimiento que tiene lugar, que se encuentra en el filo de ese metal que traspasa la carne ofrecida y en el grito del sacrificado. La tragedia, sin embargo, no tendría lugar ahí en ese acto, la verdadera tragedia, por parafrasear a Mesguich, sólo sería posible en el teatro.

La segunda tesis establece una gran diferencia entre el teatro y el sacrificio, no se pueden identificar ni uno incorpora al otro. Si habíamos considerado al pensamiento filosófico como el ejecutor del sacrificio, del fin de la tragedia, ahora aparece el teatro como el que acaba sacrificando al sacrificio, mientras que 
en la filosofía parecería quedar algo del mismo. El teatro es consciente de que el sacrificio ofrecido es sólo un acto fingido:

Esta suspensión del sacrificio, esta puesta en escena en el lugar de eso que tiene lugar, supone una extraña institución, que a la vez asegura la puesta en escena, se pone ella misma en escena y se desinstitucionaliza, cada vez, cada día, a cada estreno. (Derrida, 1993, 58)

Con ello observamos un nuevo aspecto: el de la institución. El teatro pone en guardia contra la institución al mismo tiempo que la guarda. Por ello, la institución está aliada con la memoria, con lo que la memoria guarda, por eso la institución representa lo inmóvil, una retención del tiempo, supone fosilización. Esta suplementariedad del sacrificio de la que derivaría esta institucionalidad pasajera o provisional que ha de renovarse en cada representación está ligada a esa distinción entre tener lugar y poner en escena. Es el juego de estos acontecimientos -y la memoria de éstos- lo que vendría a determinar la institucionalización y no el sacrificio, que aquí es un elemento externo. Pero, ¿̨es suficiente este desplazamiento tópico del sacrificio para suspenderlo?

Podemos definir el sacrificio como un acto ritual de purificación. Se ofrece a los dioses para calmar su ira, para apaciguarlos y así restituir el orden. El sacrificio es un acto social mediante el cual la comunidad espera sobrevivir. Por eso el sacrificio ofrece las dos caras, la de la destrucción y la de la salvación, en un único acto. Y si el sacrificio es constitutivo de la tragedia - no sólo de la griega- y del teatro, es porque en él mismo se encuentra reflejada la contradicción: es un acto de muerte que da vida, es un acto personal y social al mismo tiempo; es un acto de sumisión y de sublevación. Sin esta paradójica esencia del sacrificio no habría tragedia. Podemos afirmar así, con María Zambrano (1989), que:

(...) el conflicto trágico no alcanzaría a serlo, a ingresar en la categoría de la tragedia, si consistiera solamente en una destrucción; si de la destrucción no se desprendiera algo que la sobrepasa, que la rescata. Y de no suceder así, la Tragedia sería nada más que el relato de una catástrofe o de una serie de ellas, en la cual, a lo más se ejemplifica el hundimiento de un aspecto de la condición humana o de toda ella. (18)

Antígona se nos presenta como la perfecta víctima de sacrificio. Joven doncella inmaculada que ha de pagar con su vida un tributo a los dioses. Antígona arrastra la mancha no purificada aún de su origen. Su padre Edipo, el más 
desgraciado de los hombres, cumplió su fatal destino, del cual sus hijos son el testimonio viviente. Los hermanos varones de Antígona cumplen con su destino trágico dándose muerte uno a otro -la mejor metáfora para una guerra civil-y Antígona ha de cumplir el suyo. Creonte, el tirano de Tebas, sucesor de Edipo, hermano de Yocasta (que es la madre de Antígona y también de Edipo), ordena que el cadáver de Policines, el hermano traidor a su ciudad, no reciba enterramiento. Antígona es llevada a la muerte por su familia, a causa de esta marańa inevitable que le ha hecho ser lo que es. Ella, Antígona, fiel a los dioses frente a las leyes de la ciudad, no reconoce la autoridad del gobernante, sino la fidelidad a su estirpe, que va más allá de las leyes de los hombres e incluso de los dioses. Porque a Antígona su sacrificio la libera de la vida, una vida que no es vida, como ya le anunció su padre desde que conoció su origen y su crimen. Antígona no podía librarse de su destino, pero con su sacrificio libera a su hermana Ismene, limpia su culpa, que no es otra que haber nacido. Antígona sacrifica su vida, y con su sacrificio ella también recibe vida, la que no podía de otra manera tener. $\mathrm{Su}$ acto la hace digna de los dioses. Su acto borrará la mancha que arrastra, aunque nadie la señale. La mancha terrible de una feroz endogamia que consumiría a la ciudad. Con su sacrificio Antígona evita su boda con Hemón, hijo de Creonte y por tanto primo de Antígona. Su sacrificio honra a su hermano en nombre de una ley divina que ningún dios viene a certificar, pues éstos han desaparecido de la escena. Ningún dios interviene para mitigar o agravar su desgracia. Tampoco lo hicieron con su padre Edipo. Abandonada de la mano de los dioses, Antígona, al igual que Edipo, da cumplimiento a su destino de una forma completamente humana: sufriendo.

Pero con Antígona también tiene lugar otro desafío a la ciudad y a la ley del gobernante, ya que quien la desafía es una mujer. Antígona representa el papel que le hubiese tocado a un hombre. Pero ya no quedan hombres en su familia. Su hermana Ismene le recuerda su condición diciéndole:

Además es preciso tener presente, de un lado, que nacimos mujeres, de manera que no podremos luchar contra hombres; y de otro, que estamos obligadas por los que son más poderosos a obedecer en esto y aún en cosas más dolorosas que éstas (Sófocles, Antígona, 61-65).

Así pues, la afrenta de Antígona es doble: desafía a la ciudad y a sus leyes no dando cumplimiento a una orden expresa del gobernante y desafía a la ciudad no dando cumplimiento a una orden implícita: ninguna mujer desempeñará el lugar de un hombre. Antígona sabe de este doble desafío. Su sacrificio es tal 
porque ella sabe cuál será su destino fatal y lo acepta resignada. Para Antígona aceptar esto no es difícil tras haber tenido que aceptarse a sí misma como hija y nieta de su madre, como hija y hermana de su padre, como hermana y tía de sus hermanos. La paradoja de su origen la convierte en paradoja viviente.

El sacrificio de Antígona ¿ha tenido lugar alguna vez si no es con su puesta en escena? Sólo con la imagen de Antígona en su tumba, enterrada viva en una gruta, no hay sacrificio ni tragedia. Habría la mostración de una muerte, de una forma terrible y agónica de morir. Pero no tragedia ni sacrificio. El sacrificio tiene lugar entonces en el enfrentamiento de la ley, en todos los aspectos que se van mostrando en escena y que hacen que Antígona sea una tragedia. El sacrificio sólo tiene lugar en escena, con su puesta en escena en la Tragedia. El sacrificio de Antígona también representa el de la figura de la mujer en el pensamiento occidental. El falogocentrismo ha enterrado en la gruta ${ }^{3}$ de la neutralidad del ser humano a la diferencia sexual, a la diferencia sexual como mujer. $Y$ al mismo tiempo, ha reprimido este acontecimiento, lo ha olvidado, lo ha excluido de la historia.

\section{La represión}

Otra manera de pensar lo irrepresentable en el teatro tiene que ver con el concepto psicológico, psicoanalítico -y también político- de represión. Derrida señala que Mesguich vendría a proponer, en su teatro de la paradoja, un teatro de la represión.

Esta puesta en escena de la represión no es un simple levantamiento de la represión, una simple liberación, una puesta al desnudo de esto que es

\footnotetext{
${ }^{3}$ La figura de Antígona toma cuerpo en Glas (Derrida, 1974), al hilo de la madeja familiar de Hegel y de las referencias de éste último a la protagonista de la tragedia de Sófocles. Respecto a lo que venimos desarrollando como lo excluido, lo reprimido o lo invisible, podemos leer en la citada obra:

A nosotros, como a Hegel, nos ha fascinado Antígona, esa increíble relación, esa poderosa ligazón sin deseo, ese inmenso deseo imposible que no podía vivir, capaz tan solo de invertir, paralizar o exceder a un sistema y a una historia, de interrumpir la vida del concepto, de cortarle el resuello o, lo que viene a ser lo mismo, de soportarlo desde el afuera o el fondo de una cripta.

Cripta -se diría de lo trascendental o de lo reprimido, de lo impensado o de lo excluido- que organiza el suelo al que no pertenece. (187a) [La $a$ indica la columna de Hegel]. Traducción española coordinada por Cristina de Peretti y Luis Ferrero (2015).
} 
impresentable. Se trata de una presentación paradójica de lo impresentable «como tal». El «como tal» fenomenológico debe ser aquí afectado de una modificación esencial. $(1993,60)$

Respecto a la represión podemos decir, en términos psicoanalíticos, que ésta es ya una representación. Freud en «La represión» describe este mecanismo del siguiente modo: "Su esencia consiste exclusivamente en rechazar y mantener alejados de lo consciente a determinados elementos» $(1993,647)$. Esta definición psicoanalítica de la represión puede extenderse al ámbito político. La represión política también consiste en rechazar y mantener alejados a ciertos elementos. Sin embargo hay diferencias entre el concepto psicoanalítico de represión y el político, aunque ambos puedan ser equiparados en su definición. La represión psicoanalítica siempre es represión de una representación. Así lo describe Freud en «Lo inconsciente» (1993):

Una pulsión no puede devenir nunca objeto de la conciencia. Únicamente puede serlo la idea que la representa. Pero tampoco en lo consciente puede hallarse representada más que por una idea. Si la pulsión no se enlazara a una idea ni se manifestase como un estado afectivo, nada podríamos saber de ella. Así pues, cuando empleando una expresión inexacta hablamos de mociones pulsionales, inconscientes o reprimidas, no nos referimos sino a mociones pulsionales, cuya representación ideológica es inconsciente. (198)

Luego, la puesta en escena de la represión sería la representación de una representación que nunca está presente. Pues la represión, para que sea tal, ha de permanecer en el inconsciente. A toda represión corresponde una resistencia, una fuerza contraria en el consciente que se opone a ella e impide que lo reprimido aflore. No hay puesta en escena de la represión psicoanalítica. En todo caso, habría puesta en escena de la resistencia. De este modo, el mecanismo de la represión, la representación de una pulsión reprimida, seguiría quedando invisible. Pero es cierto que no hay levantamiento de la represión por su revelación. La represión sigue funcionando aunque se descubra lo reprimido. En ese sentido, la represión sigue siendo invisible. El individuo que sufre la represión es ciego a ella, y ni siquiera delante de sus ojos es capaz de verla. Pero la dificultad de ver la represión no es sólo para el que la padece, ya que si el mecanismo de represión funciona bien ésta es invisible. Sólo los fracasos de la represión son observables, y sólo a través de ellos se puede analizar. Por lo tanto, la represión, cuando funciona, es doblemente invisible. 
Evidentemente, si se destaca el mecanismo de la represión como aquí se hace, se está aceptando la separación psicoanalítica entre consciente/inconsciente, y esta separación está trabajando todo el texto. La palabra invisible es así la palabra inconsciente. Los pares consciente/visible e inconsciente/invisible pueden complicarse aún en la metáfora cognoscitiva del ojo convirtiéndose en consciente/visible/cognoscible e inconsciente/invisible/incognoscible. La filosofía generalmente ha desechado todos los aspectos que no son asimilables al primer trío de conceptos. Incluso la misma separación psicoanalítica entre consciente/ inconsciente fue problemática. Es en este sentido en el que Derrida se pregunta en "Tímpano» (1994) (una vez realizado un análisis deconstructivo que sitúa al tímpano en una encrucijada entre lo natural y lo artificial) lo siguiente:

¿Diremos desde este momento que lo que aquí resiste es lo impensado, lo reprimido, lo rechazado de la filosofía? Para no agarrarse más, como a menudo se hace hoy, a la equivalencia confusa de estas tres nociones, una elaboración conceptual debe introducir ahí un nuevo juego de la oposición, de la articulación, de la diferencia. Introducción, pues, a la différance. (34)

Desde este punto de vista, parece que sería necesario introducir en el sugerente texto de Mesguich este «nuevo juego de la oposición», pues, en lo que atañe a la represión y al sacrificio, aún parece prevalecer en él la distinción fundamental entre realidad y ficción. Sólo desde esta distinción puede entenderse que el sacrificio quede suspendido en el teatro. Mesguich (1991) escribe la escena:

El actor no como víctima expiatoria, chivo expiatorio, sino como aquel que representa (joue) la víctima; que juega, delante de todo el mundo, con la ley. Aquel que finge (representa/remeda al) ser chivo. En el teatro, al final, Abraham $^{4}$ y el cordero se levantan y saludan. (Derrida, 1993, 58)

La conciencia de simulacro de este actor recorta las posibilidades deconstructivas del simulacro que, evidentemente, no está funcionando en este texto. Si los efectos deconstructivos del simulacro hacen impertinente la oposición ficción/ realidad, presencia/ausencia, etc..., los efectos de este fingimiento consciente

\footnotetext{
${ }^{4}$ Es interesante, a propósito de Abraham, señalar aquí la reflexión que Derrida realiza en Donner la mort, sobre todo en el trabajo La litterature au sécret. Une filiation imposible, (1999) acerca del sacrificio como origen y posibilidad de la literatura. La reflexión surge a propósito de las posibles lecturas del sacrificio de Abraham, sobre la relación padre/hijo, relación doblada, Dios/Abraham, Abraham/Isaac.
} 
acentúan aún más esta oposición. La representación vuelve pues a ser repetición, copia, de un acto presente, de una presencia que es la que lleva impresa la característica de real.

La separación consciente/inconsciente no trabajaría sólo el texto propuesto, sino que recorrería otros textos, pues ésta vendría a representar también la escena de la vida psíquica. Nos dice María Zambrano en la obra ya citada (1989):

Antígona en su tumba es una presencia. En la vida común la persona en el mejor de los casos, llega a hacer esa su máscara un tanto transparente y al par animada, pues que no hay que olvidar que de luz de vida estamos tratando. Mas en la vida de una persona humana, por dada que sea a la luz, hay siempre una oscuridad y en ella algo que se esconde; la persona resiste a la luz en los mejores casos tanto como la busca. Sólo por el sacrificio se deshace esta resistencia - sacrificio no visible en muchos casos y en otros, cumplido en instante violenta y visiblemente, mas incubado desde un principio. (37)

Reconocimiento de una estructura psíquica en la que la oscuridad (el inconsciente) siempre está trabajando. Y en la que la resistencia se nos ofrece como el elemento que equilibra la balanza de la vida. El sacrificio aparece aquí como un elemento rescatador, como la superación de la represión, que en el acto consciente o no del sacrificio sucedería de golpe. La víctima del sacrificio, incluso aunque sea víctima de sí misma, ante esa situación final deja de resistir. ¿¿Puede tal afirmación mantenerse en un contexto psicoanalítico? ¿Y en el político?

\section{El tiempo}

Otro aspecto interesante en la obra de Mesguich es su caracterización del tiempo teatral. El teatro tendría "por esencia una cierta repetición». Una repetición que hace que cada vez sea la primera, y esta presencia, este presente único sólo puede surgir a través de esa estructura de repetición. La repetición no imita, no copia: abre camino a un nuevo y único instante y con él a una temporalidad nueva, diferente. La temporalidad teatral descrita por Mesguich vendría a considerar un presente no regulado por el presente: «Pensar sobre el escenario significa este increíble espacio donde el saber no puede decidir de esto qué es el presente» (Derrida, 1993, 62-63). 
Pensar sobre el escenario. Pensar encima del escenario. Pensar en el escenario. Pensar el escenario. Pensar acerca del escenario. Primero decidir qué pensar, dónde situarse en este verdaderamente increíble espacio -el escenario- que representa también otro espacio, que está siendo puesto en escena al mismo tiempo. Un espacio que no es decidible y un tiempo que carece de presente rector, pero que se constituye a través de múltiples presentes siempre diferentes y siempre el mismo. La temporalidad teatral carece de memoria si el teatro es entendido en términos clásicos: si el teatro es re-presentación. Y el tratamiento temporal de Mesguich ¿continúa anclado en el esquema tradicional: autor-actor-público? Si así fuese ¡qué lejos de Artaud!' Porque a pesar de esa estructura de repetición inaugural ¿mantendría el teatro que entrevemos de Mesguich la fidelidad a un texto al que presenta (no re-presenta) cada vez, cada función?

Mesguich juega continuamente con distintas perspectivas. Unas veces se sitúa sobre el escenario como el actor y nos dice cómo ve el teatro el actor; otras es el público y otras el director de escena. Y es entre bambalinas donde Derrida encuentra nuevamente otra formulación del tiempo teatral. Es la prisa del actor, la precipitación, su rapidez aquello que conduce hacia la categoría de furtivo:

(...) la categoría de furtivo o de clandestino significa que el instante esencial del teatro no se deja integrar en la temporalidad general, está robado al tiempo, y este es también un momento de presentación de la ley y por tanto de la transgresión de la ley. Es un momento anormal, que expone la ley como represión. (63)

Esta dinámica de apresuramiento del teatro, de los actores, en la que se produce una transgresión temporal (y que representa al mismo tiempo la ley y su transgresión) es opuesta a la filosofía, en la cual, parecería que ese apresuramiento teatral se transforma en una paciente y sosegada reflexión. Es aquí donde Derrida, entre estas dos hipótesis, introduce esos "golpes de efecto» (coups de théatre) en la filosofía. Golpes de efecto que, por otra parte, no necesitan ser introducidos, sino más bien, reconocidos como tales. «Hay en el pensamiento filosófico

\footnotetext{
${ }^{5}$ Derrida, J. (1989): "El teatro de la crueldad y la clausura de la representación". La escritura y la diferencia. Barcelona: Editorial Anthropos.

«El teatro de la crueldad no es una representación. Es la vida misma en lo que ésta tiene de irrepresentable. La vida es el origen no representable de la representación», (320).

También nos dice Derrida de Artaud que: «...es al teatro como repetición a lo que no puede resignarse, el teatro como no-repetición a lo que no puede renunciar», (342).
} 
pre-institucional, instantes que se parecen a esta urgencia furtiva, clandestina, no autorizada y loca, que ponen a la filosofía en margen» (64).

Y quizá se trate de eso, de pensar ese tiempo desquiciado y loco (el tiempo del espectro) para que el pensamiento filosófico, en el margen de sí mismo, pueda pensar de otro modo, pueda pensar a destiempo.

\section{Conclusiones}

Derrida nos ha ofrecido una reflexión crítica en el contexto de la representación teatral que nos obliga a leer entre líneas, a tratar de pensar aquello que permanece aún invisible en el seno de la representación que el teatro nos muestra. Y aquello que la representación teatral nos ofrece sobre la representación misma y sobre el teatro mismo no se circunscribe únicamente al ámbito del teatro o de las artes escénicas sino que supone también un cuestionamiento de la filosofía, del concepto de representación articulado por el pensamiento occidental. La representación teatral que se ofrece en este texto es al mismo tiempo la puesta en escena de la filosofía, y el argumento de esta representación, como hemos observado (seguimos en el ámbito de la metáfora cognoscitiva del ojo), se nos ofrece en varios actos tratando de mostrar aquello que no se puede representar, que se escapa, que elude la representación. Ocultar lo irrepresentable de la representación en la representación, dentro de ella misma: el sueño de la metafísica. De aquello que escapa a esta representación su ocultamiento se realiza como parte de un programa que viene a excluir sistemáticamente eso irrepresentable del ámbito no sólo de lo no cognoscible sino incluso del ámbito de lo pensable. Lo impensable, lo invisible, lo imposible: estas son algunas de las tareas que la deconstrucción de Derrida ha dejado como reto al pensamiento filosófico contemporáneo.

\section{Bibliografía}

Derrida, J. (1993). "Le sacrifice". La Méthaphore, No. 1, Printemps, pp. 51-65.

(1974). Glas. Paris: Galilée. Trad. española coordinada por Cristina de Peretti y Luis Ferrero (2015). Madrid: La Oficina Ediciones.

(1987). "Le retrait de la méthaphore". Psyché. Inventions de l'autre. Paris: Galilée, pp. 63-94.

(1987). "Envoi”. Psyché. Inventions de l'autre. Paris: Galilée, pp. 109-144. 
(1989). "El teatro de la crueldad y la clausura de la representación". La escritura y la diferencia, Barcelona: Editorial Anthropos, pp. 318-343.

(1994). "Tímpano". Márgenes de la Filosofia. Madrid: Cátedra, pp.15-36.

(1990). Mémoires d'aveugle. L'autoportrait et autres ruines. Louvre, Réunion des Musées Nationaux.

(1999). Donner la mort. Paris: Galilée.

(2013). Penser à ne pas voir - Ecrits sur les arts du visible (1970-2004). Paris: Editions de la Différence.

Freud, S. (1993). Los textos fundamentales del psicoanálisis. Barcelona: Altaya.

Mesguich, D. (1991). L'éternel éphémère, Paris: Le Seuil.

Platón (1999). La República o el Estado. Madrid: Espasa-Calpe.

Sófocles (2005). Ayax. Las Traquinias. Antígona. Edipo Rey. Madrid: Alianza Editorial.

Zambrano, M. (1989). La tumba de Antigona. Madrid: Mondadori.

Recibido : 05/10/2015

Aceptado : 23/10/2015

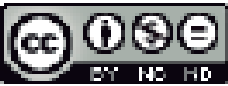

ENDOXA está bajo una licencia de Creative Commons Reconocimiento-NoComercial-SinObraDerivada 4.0 Internacional 